\title{
EL NEUROMARKETING Y LA INGENIERÍA DEL BRANDING
}

NEUROMARKETING AND BRANDING ENGINEERING

Jorge Arroyo Prado ${ }^{1}$

\section{RESUMEN}

Neuromarketing es el estudio del funcionamiento del cerebro en las decisiones de compra de un producto, es decir, la manera en que las personas eligen. Es un nuevo campo del marketing que investiga la respuesta cerebral a los estímulos publicitarios, de marca y de otro tipo de mensajes culturales. Intenta buscar la clave de la decisión de compra que todos tenemos en el cerebro. La ingeniería del branding tiene como objetivo mantener en el cerebro de las personas lo que ha visto y escuchado intensamente, de forma que se establezca un recordatorio mental. Por esto las empresas utilizan estrategias que generan recordatorios de sus marcas para impulsarlos a la compra.

Palabras clave: neuromarketing, grupos de enfoque, resonancia magnética nuclear, encefalografía, tomografía, seguimiento de los ojos, ingeniería del branding, brand equity.

\begin{abstract}
Neuromarketing is the study of brain functioning regarding decisions undertaken to buy a product; that is, it is the study of how people choose. It is a new field of marketing which researches the brain's response to stimuli advertising, branding and other cultural messages. Try to find keys to purchasing decision we all have in the brain. Branding Engineering aims to keep in people's mind what they have seen and listened intensively. That is why companies use strategies which generate reminders of their brands to encourage people to purchase them.
\end{abstract}

Keywords: neuromarketing, focus group, functional magnetic resonance, magneto encephalography, tomography, eye tracking, branding, brand equity engineering.

1 Doctor en Administración, Magíster en Administración con mención en Gestión Empresarial, Ingeniero Químico. Profesor principal de la Universidad Ricardo Palma, Decano de la Facultad de Ingeniería, Av. Benavides s/n, Lima, Perú. <jarroyo@urp.pe> 


\section{DESCRIPCIÓN}

El comportamiento del consumidor en la compra de productos o servicios siempre ha sido una de las preocupaciones de los especialistas en marketing. Su concepción siempre consistió en que los cambios en el comportamiento del consumidor provienen de los estímulos que reciben, especialmente de estímulos externos. Estos se concretan en el cerebro del consumidor, a través de un proceso de toma de decisiones que es cambiante y en el que influyen factores culturales, económicos, sociales, valores individuales y psicológicos. Philip Kotler y otros investigadores manifiestan que el cerebro se comporta como una caja negra donde se acumulan todos los estímulos externos que recibe del marketing de las empresas que están en su entorno y que estos, de una u otra forma, logran mantenerse en la mente de los consumidores. Es decir, las marcas logran posicionarse de tal modo que, en el momento que los consumidores demanden una necesidad, recurran a la compra de sus productos, debido a que las mantienen almacenadas en sus cerebros.

Sin embargo, los procesos de continuación de compra dependen del valor que perciben del producto. Por tal razón los especialistas en marketing llevan a cabo una serie de estudios y pruebas, que incluyen encuestas, focus group, métodos de prospectivas y escenarios, testeo de productos, y otros, para conocer a los consumidores y lograr, mediante estas y otras estrategias adecuadas de marketing, diferenciar sus productos respecto a los de la competencia. Con ello, logran el posicionamiento de los productos en la mente de los consumidores y su fidelización.

Los especialistas en marketing desean saber con un buen nivel de certeza la respuesta a estas interrogantes: ¿qué debe contener un comercial para lograr un mayor impacto en el consumidor?, ¿qué elementos se debe investigar en el mercado objetivo para conocer con precisión lo que sienten y piensan los consumidores?, ¿cuál debe ser el grado de repetición en los medios de publicidad para que una campaña sea efectiva?, ¿qué estímulos sensoriales debe contener un producto para lograr dar valor y satisfacción al cliente?, ¿̨cuál debe ser el precio razonable para que decidan la compra?, ¿qué

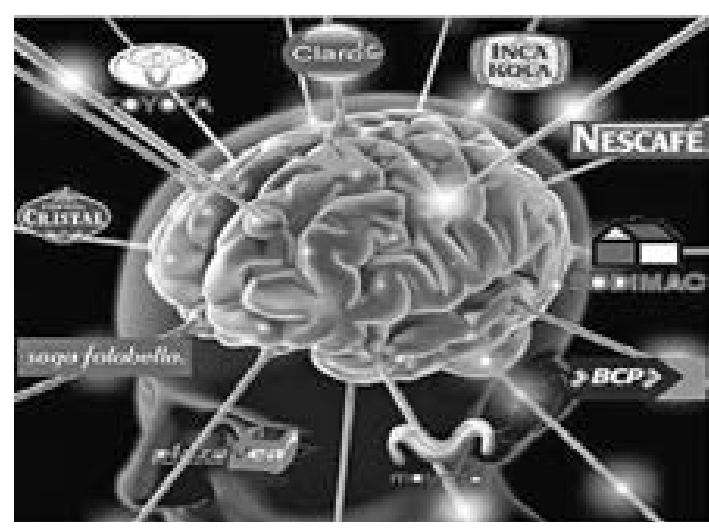

Figura 1: El neuromarketing es el estudio de los procesos cerebrales, los cambios en los procedimientos de toma de decisiones y en las compras de los productos. se debe hacer para que los clientes se sientan satisfechos y regresen a comprar?, entre otras. Todas estas preguntas las ha tratado de responder, a partir de la neurociencia, el neuromarketing.

A fines del siglo pasado se marca un hito en la historia con el surgimiento de la neurociencia, a partir de la cual se crea una nueva disciplina llamada neuromarketing, que consiste en el estudio de los procesos cerebrales y sus cambios en los procedimientos de toma de decisiones en las compras de los productos. Las empresas se han dado cuenta que esta disciplina es muy importante porque pueden sacar conclusiones útiles de esta herramienta, aunque todavía no ha alcanzado todo su potencial. Por ejemplo, a las empresas les interesa saber qué es lo que determina las compras del consumidor. En otras palabras, qué factores influyen en ellos y cómo pueden influenciar la iluminación, el sonido, los colores, el olor, el sabor, el peso, el volumen, es decir, todo aquello que sensorialmente interviene en el proceso de la compra, ya que el primer contacto que tiene el consumidor con el producto es la vista y los sentidos. Para obtener respuesta a esta inquietud, desde el punto 
de vista del neuromarketing, es necesario escanear el cerebro e interpretar los datos que se obtienen en el monitor.

Las técnicas que utiliza el neuromarketing para medir la atención, la emoción y la memoria son cuatro:

- La resonancia magnética funcional (FMRI): monitorea funciones fisiológicas.

- La encefalografía (EGG): mide los cambios eléctricos en el cerebro

- La magneto encefalografía (MEG): mide los cambios magnéticos que se producen en el cerebro.

- La tomografía (PET): monitorea las funciones fisiológicas de las alteraciones que pueden surgir con la actividad cerebral.

Existen otras técnicas que también se utilizan en el neuromarketing, tales como:

- El eye tracking: consiste en el seguimiento de los ojos. Se evalúa el punto donde se fija la mirada y el movimiento de la cabeza.

- La electromiografía (EMG): es una técnica para la evaluación y registro de la actividad eléctrica producida por los músculos esqueléticos.

- El control del ritmo cardiaco: este se acelera cuando estamos ante una situación emocionante o estresante. También indica que algo nos pone nerviosos para bien o para mal.

- La respuesta galvánica de la piel: mide el miedo, la ira o los sentimientos sexuales, a través de los cambios en la resistencia eléctrica de la piel.

La herramienta más usada es la resonancia magnética (FMRI), una técnica no invasiva que utiliza la resonancia de los campos magnéticos, por medio de la cual se obtiene información sobre la estructura y áreas del cerebro que se activan ante ciertos estímulos o situaciones determinadas, como son la publicidad de ciertos productos o el nombre de una marca.

\section{EL CASO COCA COLA}

Una de las pruebas de neuromarketing más citadas fue la llamada "Desafío Pepsi” en la que a un grupo de personas se les invitó a probar dos bebidas gaseosas que no mostraban diferencias a simple vista. A ellas se les hizo un monitoreo de su actividad cerebral, mediante resonancia magnética, y se encontró que más del 50\% preferían Pepsi en vez de Coca Cola, a pesar de que Pepsi solamente tenía el 25\% del mercado de las bebidas gaseosas.

Read Montagne, especialista en neurociencia, repitió el experimento, pero esta vez se les hizo conocer previamente la marca, lo que invirtió el resultado, puesto que el $75 \%$ de las personas escogieron Coca Cola. La zona responsable de la recompensa positiva del cerebro se activaba con ambas gaseosas, no obstante, el córtex prefrontal medio también se activó al conocer la marca, lo que evidencia lo racional e irracional que es la mente humana al realizar procesos de decisión de compra. Con esto se determinó que las personas, en la mayoría de las ocasiones, no compran de forma racional y por lo tanto es de gran importancia la publicidad y el posicionamiento de marca en la mente del consumidor. Esto confirmó, además, que el componente emocional dentro del proceso de compra es muy alto, lo cual era casi imposible de evidenciar con las

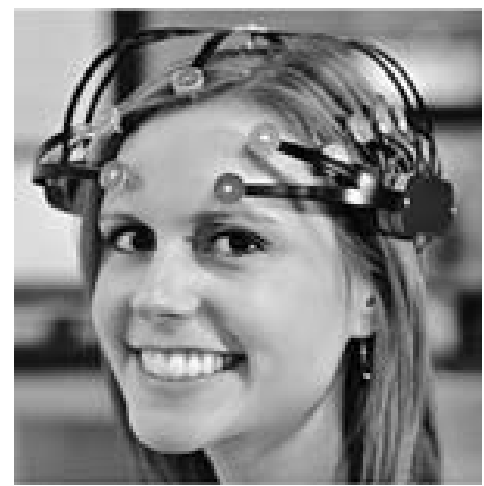

Figura 2: Técnicas usadas en el neuromarketing 
metodologías clásicas, pues las respuestas que el consumidor o cliente proporcionaba, eran racionales y no emocionales. Este experimento reconoció al neuromarketing como una disciplina importantísima, ya que los cerebros reconocían la marca de acuerdo al comportamiento del mercado. Así, cada día, con las nuevas técnicas de la neurociencia, se configura el neuromarketing como una herramienta fundamental para el ámbito de la mercadotecnia.

Es indudable que el cerebro es el órgano humano en el cual se puede encontrar toda la información que buscan las empresas. Cuando se activa la zona de atención, emoción y memoria ante los estímulos recibidos de un producto o servicio se puede comprobar la efectividad de un anuncio o comunicación. En este sentido, el neuromarketing permitiría mejorar las técnicas de publicidad, así como mejorar la comprensión de la relación entre la mente y la conducta del consumidor, lo cual siempre ha sido el desafío más importante del marketing. Según Le Monde, se trataría de la última versión de la percepción subliminal, que trataría de impregnar el cerebro de publicidad, sin que las personas puedan darse cuenta. Los detractores de esta disciplina critican que se podría llegar a controlar y enfocar las decisiones de consumo de los clientes hacia productos o servicios de mala calidad. Por el contrario, Braidot, especialista en el tema, indica que el neuromarketing ofrece un conjunto de recursos de enorme valor para investigar el mercado, segmentarlo y desarrollar estrategias exitosas en materia de productos, tales como diseño, marca (branding), packaging, posicionamiento, precios, mix de comunicaciones, canales y ventas.

El profesor Roberto Álvarez del Blanco, investigador español que visitó el Perú, y que es considerado autoridad mundial en el tema, expresa, sin embargo, que el neuromarketing no analiza a la persona como lo hace el marketing tradicional, sino que estudia el cerebro de manera rigurosa, con el fin de pronosticar el comportamiento de la persona de forma más certera. No crea necesidades sino que las satisface. Esta disciplina afirma que somos básicamente seres emocionales, ya que casi el $95 \%$ de una decisión es emocional y sólo el $5 \%$ es racional. El neuromarketing descubre lo que en neurociencias se llama el "punto $S$ " del cerebro. En este todo entra en equilibrio, en el que se han disipado riesgos e incertidumbres; desde ese momento el cerebro está listo para operar positivamente en función de una propuesta para atraer al cliente con una oferta atractiva. No obstante, el neuromarketing no controla las decisiones de compra, tan solo estimula, provoca, anima, entusiasma e ilusiona, y no se relaciona con la publicidad subliminal, que se basa en imágenes que no son percibidas por el ojo humano pero sí por el subconsciente, las cuales generan recordación (este tipo de publicidad es ilícita en algunos países). Se dice que el neuromarketing ayudará al marketing social, por ejemplo, a solucionar el consumo mortal de comida chatarra, a disminuir el consumo de tabaco, las drogas y el alcohol, además que aportará soluciones a la conceptualización de los productos. El profesor Álvarez advierte, sin embargo, que los profesionales que utilizan el neuromarketing deben actuar con códigos deontológicos muy estrictos para darle buena práctica y no utilizarlo para otros fines.

\section{LA INGENIERIA DEL BRANDING (BRAND EQUITY)}

Si de acuerdo a las investigaciones actuales, el neuromarketing no controla ni influye en las decisiones de compra, de una u otra forma sí es una manera de comprobar la enorme influencia de la ingeniería del branding, cuyo objetivo es retener en el cerebro de las personas lo que ha visto y escuchado intensamente, lo cual se mantiene en la mente como un recordatorio. Por ello las empresas utilizan estrategias que generan recordatorios de sus marcas para impulsar a las personas a la compra.

Branding es un anglicismo muy usado en mercadotecnia, que no es sino la ingeniería del proceso de diseñar y construir una marca (en inglés, brand equity) y mantenerla, mediante la planificación y 
administración estratégica del conjunto total de activos vinculados en forma directa o indirecta a un nombre y/o símbolo (logotipo) de un producto, de una empresa o incluso de un país, que la persona percibe por el valor que generan y que son identificados a través de su marca. Si se trata de productos, es un beneficio tanto para el cliente como para la empresa propietaria de los mismos.

Actualmente, diferenciar un producto de otro es cada vez más difícil, ya que la calidad y el valor que generan y los costes de los productos son similares. Por ello, la clave de los negocios está en "la ingeniería del branding", es decir, en el diseńo bien planificado para dar poder a la marca, que se obtiene de esta al convertirla en un elemento diferenciador y de recordatorio en la mente del consumidor. Es

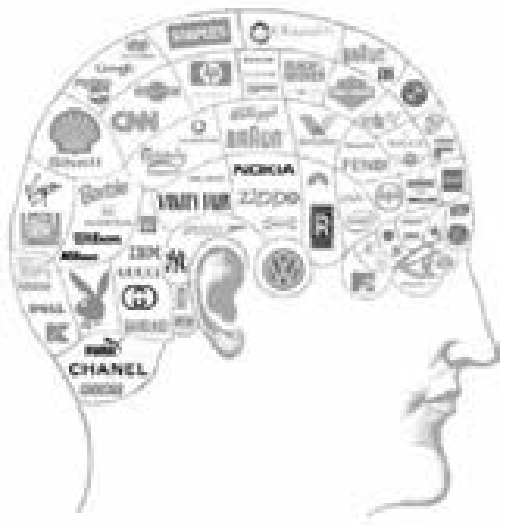

Figura 3: La marca vista como elemento diferenciador y de recordatorio en la mente del consumidor. así que las marcas tienen su propia personalidad, llamada "personalidad de marca".

Para Tom Peters, declarado "gurú de gurúes» por The Economist y la revista Fortune, lo que constituye la diferencia parte precisamente de los intangibles de una empresa: valor, credibilidad y singularidad de una marca. Así lo explica en el libro que publicó bajo el título El meollo del branding (2002). Peters demuestra que la marca es lo que define a la empresa, y que esta es mucho más que el marketing o los logotipos. Tiene que ver con la pasión, con la historia que se quiere contar, con la causa que motiva a la empresa. Las historias y experiencias serán más importantes en el futuro que los productos, afirma Peters, porque la capacidad de transmitir emoción es lo más importante en un mundo controlado por la tecnología.

Los elementos visuales de la marca son las representaciones gráficas de una empresa, que se proyectan al público a través de un símbolo o un diseńo tipográfico especial. La combinación de los elementos visuales (nombre comunicativo, símbolo, alfabeto, colores y el sistema de señalización) da la apariencia global de la empresa y constituye una expresión física muy importante en los mercados en los que ésta concurre. Es indudable entonces, como lo expresa Peters, que la capacidad de transmitir emoción es lo más importante en este mundo controlado por la tecnología.

Hoy en día se pagan grandes cantidades a los diseńadores para que creen una identidad visual fácil de recordar, pues la mayoría de los empresarios y público en general los consideran necesarios, excepto en unos pocos casos. Existen casos como el de Coca-Cola, explicado anteriormente, donde al final del experimento los consumidores la prefirieron porque su símbolo es uno de los trabajos de diseño más exitosos del mundo, y además ha sido acompañado de enormes campañas de publicidad por diversos medios, ATL y BTL para crear el posicionamiento de la marca en la mente del

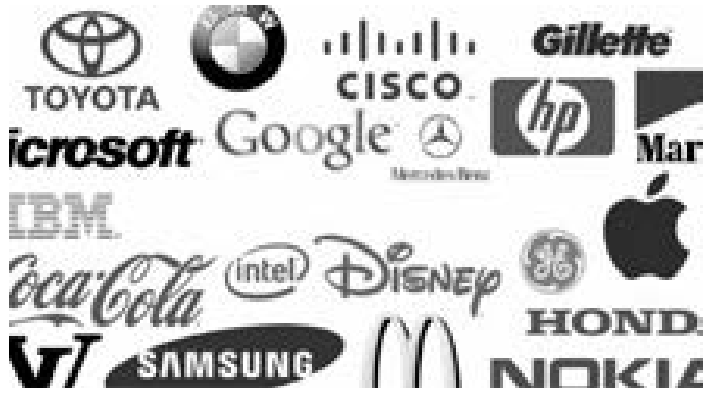

Figura 4: Según Peters, la competencia real ya no gira en torno a las cuotas del mercado, ahora se compite por la atención del cerebro y el corazón. consumidor. Por un lado, la publicidad sobre la línea (ATL, about the line) utiliza medios publicitarios convencionales masivos para posicionar una marca. Se caracteriza porque la inversión es elevada (TV, radio, periódicos, redes sociales, revistas, volantes, carteles, encartes, espectáculos deportivos etc.). Por 
el contrario, la publicidad bajo la línea (BTL, below the line) es una técnica de marketing que emplea formas no masivas dirigidas a segmentos específicos, en otras palabras, es un marketing de guerrilla que utiliza estrategias como merchadising, eventos, patrocinios, marketing directo, etc.

Según Peters la competencia real ya no gira en torno a las cuotas del mercado, sino que se compite por la atención del cerebro y el corazón, por ello, más allá del nombre de la marca, el slogan o conceptos creativos de campaña, la creación de un territorio estratégico verbal anclado en equities

\section{Ranking de marcas 2015: Top 10}

\begin{tabular}{|c|c|c|c|c|}
\hline $\begin{array}{l}\text { Ran } \\
\text { king }\end{array}$ & Marca & $\begin{array}{l}\text { Volor } \\
\text { Mules de } \\
\text { Malils s }\end{array}$ & $\begin{array}{l}\text { Sabel } \\
\text { hala }\end{array}$ & $\begin{array}{l}\text { Valoradican } \\
\text { de mances }\end{array}$ \\
\hline 1 & Apple & 128300 & $+23 \%$ & AMA \\
\hline 2 & Samsung & 81716 & $+4 \%$ & AAA- \\
\hline 3 & Google & 76683 & $+12 \%$ & AAA \\
\hline 4 & Micresoft & 67060 & $+7 \%$ & $A A A$ \\
\hline 5 & Verizon & 50843 & $+12 \%$ & AAA = \\
\hline 6 & ATBT & 58820 & $+30 \%$ & AA. \\
\hline 7 & Amazon & 56124 & $+24 \%$ & AAA - \\
\hline 8 & $\begin{array}{l}\text { General } \\
\text { Electric }\end{array}$ & 48019 & $.9 \%$ & $\Delta A+$ \\
\hline 9 & $\begin{array}{l}\text { China } \\
\text { Mobile }\end{array}$ & 47916 & $+50 \%$ & AAA- \\
\hline 10 & Walmart & 46737 & $+4 \%$ & $\mathrm{AA}+$ \\
\hline
\end{tabular}

Fuente. BrandFinance: Pub. Er Comercio 01 de marso 2015

Cuadro 1: Top 10 del ranking de marcas 2015. Todas están valorizadas en miles de millones de dólares

en miles de millones de dólares como se muestra en el cuadro 1.

En la actualidad, las marcas desempeñan una serie de funciones muy importantes que mejoran la vida de los consumidores e incrementan el valor financiero de las empresas. Estos activos intangibles, que pueden llegar a adquirir gran valor, se protegen mediante el registro, en el caso del Perú, en INDECOPI. El derecho de propiedad intelectual garantiza que la empresa pueda invertir con seguridad en los diseños de la marca y beneficiarse de todas las ventajas de un activo intangible tan valioso. Estas ventajas son las siguientes según Philip Kotler:

1. Mejor percepción de los resultados del producto

2. Mayor lealtad

3. Menor vulnerabilidad a las actividades de marketing de la competencia

4. Menor vulnerabilidad a las crisis de los mercados

5. Mayores márgenes

6. Mayor rigidez en la respuesta de los consumidores ante los aumentos de precio

7. Mayor elasticidad en la respuesta de los consumidores ante las variaciones del precio

8. Mayor cooperación y apoyo comercial

9. Mayor eficacia en las comunicaciones de marketing

10. Posibles oportunidades de concesión de licencias

11. Oportunidades adicionales de extensiones de marca. para crear una visualidad de marca única, consistente y diferenciada en el mercado.

La conquista del espacio físico se ha extendido ahora al espacio mental, las empresas además de fabricantes de productos necesitan de manera imperativa transformarse en proveedores de significados, de mensajes y promesas, relevantes y emocionales para el cliente que tienen como objetivo. Es así que las marcas son activos intangibles de las empresas, que al lograr posicionarse en la mente de las personas son valorizadas verbales y visuales es vital 


\section{MODELOS DE LA INGENIERIA DEL BRANDING}

Existen varios modelos de ingeniería del branding o brand equity, diseñados por profesores de reconocido prestigio, especialistas en marketing y empresas o agencias de publicidad como los modelos de la Agencia de Publicidad Young and Rubicam (Y\&R), el modelo de Aaker, el modelo Branz, el modelo de la Resonancia, el modelo del Estudio A-Interbrand y otros. Todos ellos están basados en lo anteriormente expuesto. Debemos recordar también que, en nuestra actual era digital, las empresas se encuentran en una etapa de transición entre la tecnología mecánica e industrial hacia el impulso a la tecnología digital. Es indudable que las actividades del marketing y el branding se han transformado por las nuevas tecnologías de la comunicación.

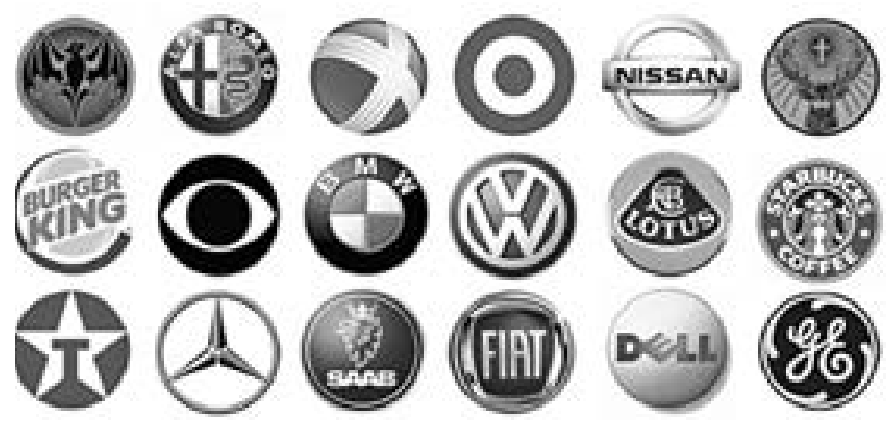

Figura 5: Estas marcas internacionales poseen gran valor como activos empresariales.

\section{CONCLUSIÓN}

El neuromarketing nace de la neurociencia y, como se ha expuesto, es actualmente la respuesta de la ingeniería del branding o brand equity, que son los esfuerzos de las empresas por crear una marca fuerte que se posicione en la mente del consumidor para la compra de sus productos. Estas marcas son activos intangibles de las empresas, que los consumidores perciben como elementos que otorgan valor y, a la vez, poseen gran valor como activos empresariales.

\section{REFERENCIAS}

Asociación de anunciantes ANDA (Abril, 2015). Branding y Diseño, la fuerza del concepto. Lima, Perú. Braidot, N. (2011). Neuromarketing Aplicado. Disponible en: www.braidot.com

García Palomo, J., Martinez Montes, E. (2013). Neuromarketing, el otro lado del marketing. Colombia:

Editorial Starbook.

Kotler, P., Keller, K. (2006). Dirección de Marketing (12ed.). México: Editorial Pearson Prentice Hall. Malfitano, C., Arteaga, R., Romano, S. (2010). Neuromarketing, Celebrando negocios y servicios.

Argentina: Editorial Granica.

Revista Business. Junio 2012, Lima, Perú.

www.ecbloguer.com

www. Markethink-consulting.com

www.es.wikipedia.org/wiki/Neuromarketing 